Article

\title{
Prognostic role of p16 overexpression in sinonasal squamous cell carcinoma: a retrospective analysis of Alberta patients
}

\author{
Jill Querney ${ }^{1,2^{*}}$, Adrian Mendez ${ }^{1,3}$, Jacob Wihlidal ${ }^{2}$, Fatemeh Ramazani ${ }^{1}$, Moses Fung1, Vincent Biron' ${ }^{1}$, and David \\ Côté1
}

1 Department of Otolaryngology - Head and Neck Surgery, University of Alberta, Edmonton, Alberta, Canada

2 Department of Anesthesiology and Perioperative Medicine, Western University, London, Ontario, Canada

3 Department of Otolaryngology - Head and Neck Surgery, Western University, London, Ontario, Canada

* Correspondence: querney@ualberta.ca

\begin{abstract}
Simple Summary: Sinonasal squamous cell carcinoma is rare amongst the general population and presents with historically poor prognosis. As a surrogate marker for human papillomavirus, p16 has been previously associated with improved prognosis and survival in squamous cell carcinoma of the oropharynx. In this study, we aim to establish correlation of p16 status with demographics, staging, treatment, and survival amonst a population-based sample comprised of patients from the Northern Alberta Head and Neck Tumour Board, Alberta Cancer Registry, and Alberta Cancer Research Biobank. Through improved understanding of pathologic factors implicated in sinonasal cancer, outcomes and prognosis can be better characterized and studied.
\end{abstract}

\begin{abstract}
Background: Sinonasal squamous cell carcinoma (SNSCC) is rare in the general population. No clear and consistent etiologic correlation between human papillomavirus and SNSCC has yet been delineated in literature. p16 is a tumour suppressor protein used as a surrogate marker for HPV. This study aims to evaluate the relationship between p16 overexpression in SNSCC and its role in prognosis and survival. Methods: A population-based retrospective analysis was performed using prospectively collected data from the Northern Alberta Head and Neck Tumour Board, Alberta Cancer Registry, and Alberta Cancer Research Biobank. p16 overexpression was analyzed from pathologic sample of patients meeting study criteria, and participants were dichotomized by status. Subsequently, nonparametric analysis of demographics, initial staging, and initial treatment were performed, and a Kapan-Meier curve was developed to assess differences in survival. Results: 16 patients were included in analysis. p16 overexpression was seen in $68.8 \%$ of patients. p16 positive and negative groups were comparable for age, gender, smoking status, stage, and treatment. A statistically significant five-year survival advantage was observed in patients with p16 positive SNSCC $(\mathrm{p}=0.013)$. Conclusions: This is the first Canadian study to demonstrate a high prevalence of $\mathrm{p} 16$ positivity in SNSCC and its presence denoting a statistically significant survival advantage. Results demonstrate a previously unconfirmed role of oncogenic HPV in SNSCC.
\end{abstract}

Keywords: Sinonasal squamous cell carcinoma; Head and neck; HPV; p16; Survival

\section{Introduction}

Squamous cell carcinoma of the nasal and paranasal sinuses is rare in the general population, with an incidence rate of $0.5-1$ per 100,000 [1,2]. While sinonasal squamous cell carcinomas (SNSCC) account for only 3\% of malignant tumors in the head and neck, there remains no clear etiologic correlation between human papillomavirus (HPV) and SNSCC [3]. Treatment of SNSCC often includes primary surgical resection followed by 
adjuvant radiation therapy [4-6]. Despite treatment, prognosis for SNSCC is poor, with a reported 5-year survival rate of $40 \%[5,6]$.

While HPV has many strains, the most prevalent oncogenic strains are HPV-16 and HPV-18 [7]. Either HPV-16 or HPV-18 have been identified in nearly $99 \%$ of cervical cancers, and viral load of oncogenic strains is a previously identified risk factor for anal and oropharyngeal squamous cell carcinomas (OPSCC) [7-10]. HPV-positive OPSCCs occur in younger patients and possess distinct molecular features associated with improved treatment response and overall prognosis [11-16].

Overexpression of may be used as a surrogate marker for high-risk HPV infection, as documented for cervical and oropharyngeal SCCs [17-19]. p16 (p16INK4a) is a tumour suppressor protein, normally repressed by the retinoblastoma protein [18-21]. However, in tumours with transcriptionally active HPV, retinoblastoma protein is inactivated, resulting in p16 overexpression $[8,22,23]$. As a result, p16 overexpression has been extensively studied as a valuable marker for oncogenic HPV infection and can be detected using immunohistochemistry.

While previous literature has reported a greater disease-free survival in HPV associated SNSCC [24,25], there is a scarcity of evidence on the association of oncogenic HPV carrier status and sinonasal squamous cell carcinoma [26]. Bishop et al. (2013) reported p16 overexpression to be an appropriate biomarker for HPV presence, in SNSCC [27].

The current study aims to examine mortality associated with p16 overexpression in SNSCC among an Alberta population-based sample. It is hypothesized that oncogenic p16 carrier status will be associated with improved prognosis, as derived from literature surrounding similar head and neck malignancies.

\section{Materials and Methods}

Prospectively collected population-based data was used to conduct a retrospective analysis of p16 expression in sinonasal squamous cell carcinoma tumors. Ethical approval was obtained from the Health Research Ethics Board of the Alberta Cancer Committee (Approval: HREBA.CC-17-0431_REN1).

In the interest of comprehensively evaluating all eligible subjects in the province of Alberta, all anonymized patient data from the Northern Alberta Head and Neck Tumour Board (NAHNTB), Alberta Cancer Registry, and Alberta Cancer Research Biobank between $2008-2017$ was examined. The NAHNTB is a multidisciplinary review board of patients with Head and Neck Cancer being treated in Northern Alberta. The Alberta Cancer Registry is a legislatively mandated and comprehensive population-based registry which records and maintains data on all cancer cases and deaths occurring in the province since 1942. The Registry records type of cancer and demographic patient information. Cancer-related deaths are recorded by the Registry using information from Alberta Vital Statistics and Statistics Canada. The Alberta Cancer Registry is operated by the Alberta Health Services - Cancer Care and is mandated by the Regional Health Authorities Act of Alberta [28]. The Alberta Cancer Research Biobank collects and stores various tumour samples from cancer patients across Alberta.

From the NAHNTB database, patients with available pathology reports were reviewed against inclusion and exclusion criteria (Table 1). Sixteen patients meeting inclusion criteria were identified and pathological evaluation was retrieved. Two researchers cross-referenced patients reports who met initial inclusion criteria, to ensure agreement of tumour origin from the sinonasal cavity and appropriate inclusion in this investigation. If there was uncertainty regarding origin of the tumour, diagnostic imaging studies were reviewed and correlated with pathology reports to determine inclusion.

Subsequently, participants were dichotomized by p16 expression status, either staining positively for overexpression, or negatively. p16 staining was considered positive when greater than $75 \%$ of the histologic sample has high-intensity, diffuse staining. p16 expression was based on third party initial pathological evaluation [29]. A Mann-Whitney 
U statistical analysis was employed for nonparametric evaluation of age, gender, smoking status, stage and treatment in the study population. A Kaplan-Meier curve was used to assess survival between the two groups. A p-value $<0.05$ was considered significant.

Table 1. Inclusion and exclusion criteria.

\begin{tabular}{ll}
\hline \multicolumn{1}{c}{ Inclusion Criteria } & \multicolumn{1}{c}{ Exclusion Criteria } \\
\hline$\bullet>18$ years of age & $\bullet<18$ years of age \\
- Biopsy proven sinonasal squamous cell & $\bullet$ Non-sinonasal malignancy \\
carcinoma & $\bullet$ No available p16 staining \\
- Available p16 staining & \\
- Registration in available datasets & \\
(NAHNTB, Alberta Cancer Registry, or Al- \\
berta Cancer Research Biobank between \\
2008-2017)
\end{tabular}

\section{Results}

\subsection{Participant Selection}

During database search for patient selection, 35 patients were originally identified with SNSCC.

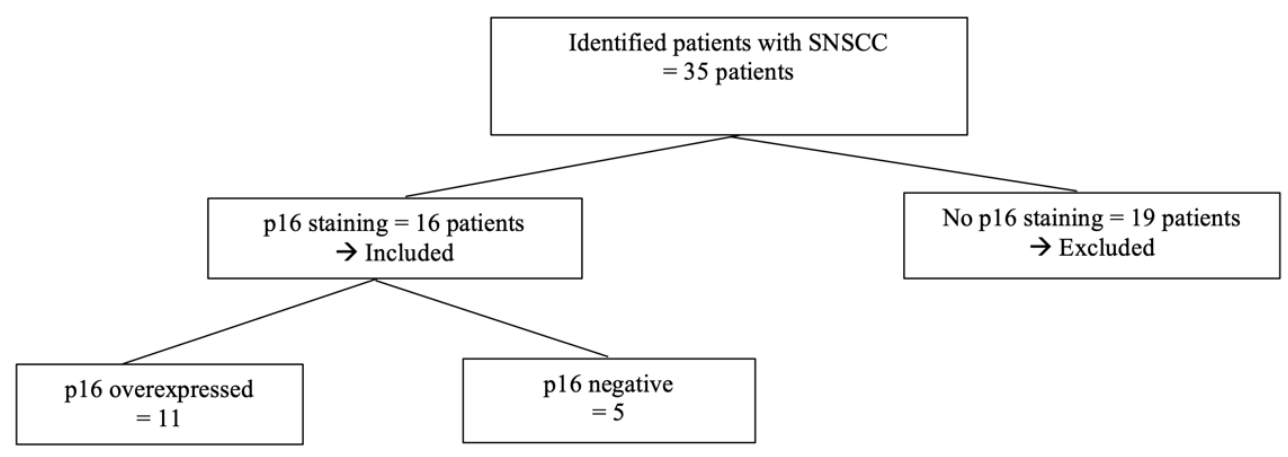

Figure 1. Patient review and study inclusion schematic.

\subsection{Demographic Analysis}

The p16 positive and negative groups were analyzed for age, gender, smoking status, stage at the time of biopsy, and initial treatment. Both groups were comparable for age, gender, smoking status, stage, and treatment $(\mathrm{p}=0.91, \mathrm{p}=0.46, \mathrm{p}=0.95, \mathrm{p}=0.32$ and $\mathrm{p}=0.17$, respectively) (see Appendix 1, Table 2).

\subsection{Survival Analysis}

All-cause mortality between the p16 positive and negative groups over 5 years was examined. Analysis of 5-year survival revealed a statistically significant increased survival rate for $\mathrm{p} 16$-overexpressing SNSCC as compared to $\mathrm{p} 16$ negative tumours $(\mathrm{p}=0.013)$. (Figure 2). 


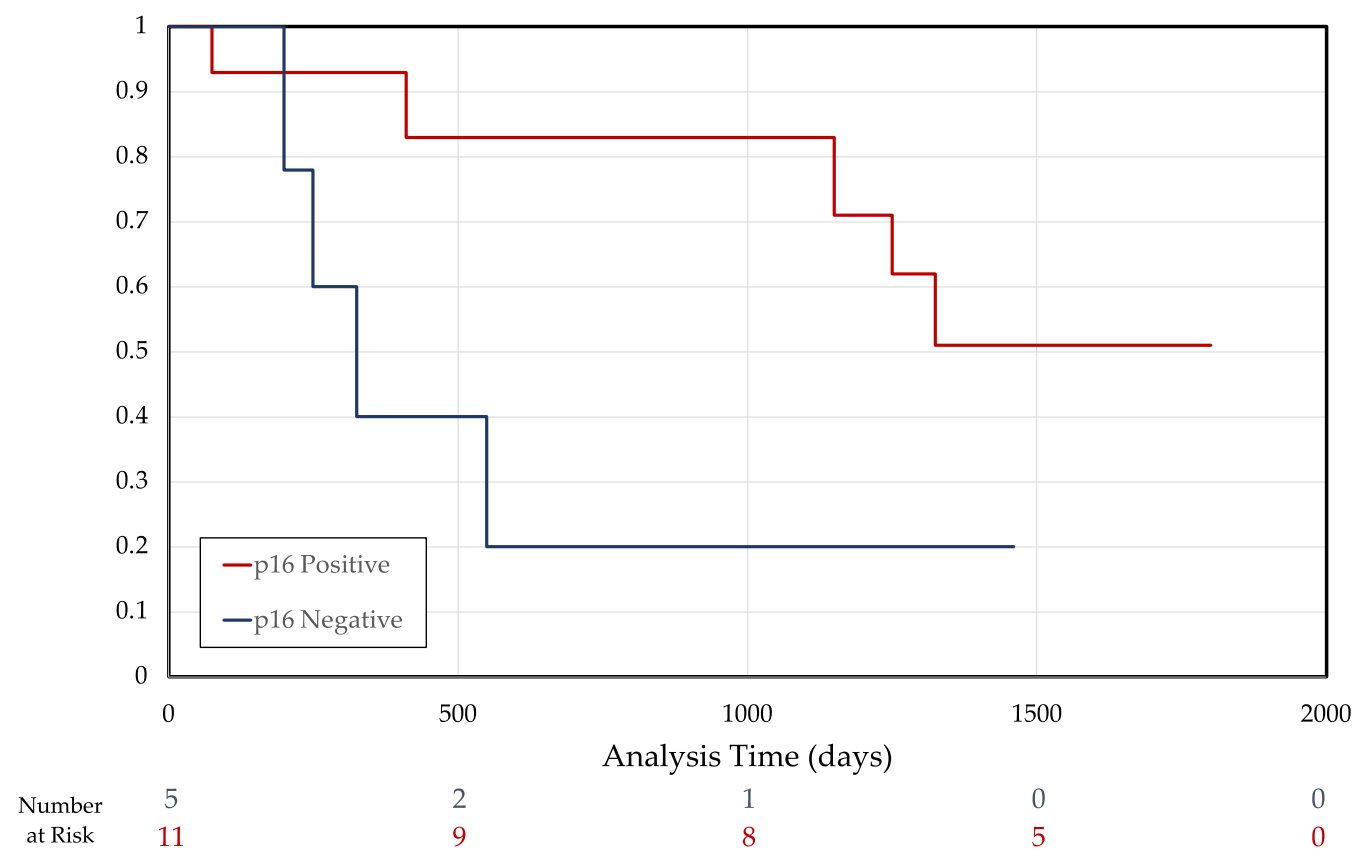

Figure 2. Kaplan-Meier survival curve of 5-year survival analysis.

\section{Discussion}

The data demonstrates a statistically significant 5 -year survival advantage in patients diagnosed with p16 overexpressing SNSCC. Analysis between the p16 positive and negative groups is comparable for age, gender, smoking status, tumour stage at the time of biopsy, and initial treatment. This is the first Canadian study to demonstrate a significant survival advantage with p16 overexpression, and therefore oncogenic HPV, in SNSCC.

Findings of this study indicate that p16 overexpressing SNSCC may behave similarly p16 overexpressed OPSCC [11-16]. This retrospective study supports further research into p16 overexpression, and HPV associated SNSCC. p16 overexpression is an adequate surrogate marker for HPV in the sinonasal cavity, supported by Bishop et al. (2013) who reported that p16 overexpression strongly correlated with the presence of HPV DNA in sinonasal tumors [27]. The current study outlines a need to delineate whether HPV-associated tumors possess distinct molecular features, as has been shown in HPV-associated OPSCC [11-16]. Further research in HPV-associated SNSCC may reveal variations in treatment response and prognosis.

Limitations of this current retrospective analysis include the small sample size, given both the limited population prevalence of SNSCC and that only tumors with p16 evaluation status were eligible for inclusion. In pathological evaluation, p16 staining was conducted at random by pathologists and without prior clinical indication. As such, there should be no inherent confounding by indication to the selection of those patients included in our analysis. Finally, the status of patient's exposure to other risk factors of SNSCC that may affect prognosis and survival was limited.

Future work will continue to validate these findings in larger patient populations, accounting for possible idiosyncrasies in this small cohort. Additionally, further molecular analysis distinguishing may reveal a pathobiological mechanism for survival differences. Finally, larger trials will characterize optimal treatment standards differentiated by p16 status, incorporating current findings as a crucial clinical parameter to guide treatment decisions.

\section{Conclusions}


This population-based study is the first in the Canadian medical literature to demonstrate the statistically significant role of p16 overexpression in prognosis and survival of SNSCC. Further work in this area to delineate the effect of HPV on treatment, prognosis, and survival for SNSCC is essential for optimal patient care.

Author Contributions: Conceptualization, Jill Querney and David Côté; Data curation, Jill Querney and Jacob Wihlidal; Formal analysis, Jill Querney; Investigation, Jill Querney and Vincent Biron; Methodology, Jill Querney and David Côté; Project administration, Jacob Wihlidal; Resources, Vincent Biron; Supervision, David Côté; Validation, Adrian Mendez and Jacob Wihlidal; Visualization, Jacob Wihlidal and Fatemeh Ramazani; Writing - original draft, Jill Querney, Jacob Wihlidal and Fatemeh Ramazani; Writing - review \& editing, Adrian Mendez, Jacob Wihlidal, Fatemeh Ramazani, Moses Fung and David Côté.

Funding: This research received no external funding.

Institutional Review Board Statement: The study was conducted in accordance with the Declaration of Helsinki and approved by the Health Research Ethics Board of the Alberta Cancer Committee (HREBA.CC-17-0431_REN1).

Informed Consent Statement: Informed consent was obtained from all subjects involved in the study.

Data Availability Statement: Not applicable.

Acknowledgments: The authorship would like to acknowledge Dr. Lakshmi Puttagunta for pathological support and input.

Conflicts of Interest: The authors declare no conflict of interest.

\section{Appendix A}

Table 2. Patient data and categorical analysis between p16 positive and p16 negative samples.

\begin{tabular}{ccccccc}
\hline ID & p16 & Age & Sex & Stage at Biopsy & Smoker & Initial Treatment \\
\hline 1 & + & 23 & F & pT1N0 & No & Surgery \\
2 & + & 70 & F & pT1Nx & No & Surgery \\
3 & + & 61 & F & pT3Nx & No & Surgery \\
4 & + & 48 & M & pT4aNx & Yes & Surgery + Adjuvant C+RT \\
5 & + & 48 & M & pT4bN0 & Yes & Surgery \\
6 & + & 50 & M & pT4aN1 & Yes & Surgery + Adjuvant RT \\
7 & + & 61 & M & pTisN0 & No & Surgery \\
8 & + & 80 & M & pT3N0 & Yes & Surgery + Adjuvant RT \\
9 & + & 69 & F & pT3Nx & Yes & Surgery \\
10 & + & 75 & M & pT4aNx & Yes & Surgery + Adjuvant RT \\
11 & + & 71 & F & pT4aNx & Yes & No treatment \\
12 & - & 55 & M & pT4N0 & Yes & Surgery + Adjuvant RT \\
13 & - & 67 & M & pT1Nx & Yes & Surgery \\
14 & - & 26 & M & pT4Nx & No & C+RT \\
15 & - & 89 & F & pT4aN2b & No & Surgery + Adjuvant RT \\
16 & - & 59 & M & T4bN1 & Yes & C+RT + Adjuvant Surgery \\
\hline
\end{tabular}

$\mathrm{C}=$ chemotherapy; $\mathrm{RT}=$ radiation therapy 


\section{References}

1. Turner, J.H.; Reh, D.D. Incidence and survival in patients with sinonasal cancer: a historical analysis of population-based data. Head Neck 2012, 34, 877-885, doi:10.1002/hed.21830.

2. Syrjanen, K.; Syrjanen, S. Detection of human papillomavirus in sinonasal carcinoma: systematic review and meta-analysis. Hum Pathol 2013, 44, 983-991, doi:10.1016/j.humpath.2012.08.017.

3. Batsakis, J.G.; Rice, D.H.; Solomon, A.R. The pathology of head and neck tumors: squamous and mucous-gland carcinomas of the nasal cavity, paranasal sinuses, and larynx, part 6. Head Neck Surg 1980, 2, 497-508, doi:10.1002/hed.2890020610.

4. Pilch, B.Z.; Bouquot, J.; Thompson, L.D.R. Squamous Cell Carcinoma. In IARC WHO Classification of Tumors, Barnes, L., Ed.; IARC Press: Lyon, France, 2005; pp. 15-17.

5. Dulguerov, P.; Allal, A.S. Nasal and paranasal sinus carcinoma: how can we continue to make progress? Curr Opin Otolaryngol Head Neck Surg 2006, 14, 67-72, doi:10.1097/01.moo.0000193177.62074.fd.

6. Dulguerov, P.; Jacobsen, M.S.; Allal, A.S.; Lehmann, W.; Calcaterra, T. Nasal and paranasal sinus carcinoma: are we making progress? A series of 220 patients and a systematic review. Cancer 2001, 92, 3012-3029, doi:10.1002/10970142(20011215)92:12<3012::aid-cncr10131>3.0.co;2-e.

7. Walboomers, J.M.; Jacobs, M.V.; Manos, M.M.; Bosch, F.X.; Kummer, J.A.; Shah, K.V.; Snijders, P.J.; Peto, J.; Meijer, C.J.; Munoz, N. Human papillomavirus is a necessary cause of invasive cervical cancer worldwide. J Pathol 1999, 189, 12-19, doi:10.1002/(SICI)1096-9896(199909)189:1<12::AID-PATH431>3.0.CO;2-F.

8. Adelstein, D.J.; Ridge, J.A.; Gillison, M.L.; Chaturvedi, A.K.; D'Souza, G.; Gravitt, P.E.; Westra, W.; Psyrri, A.; Kast, W.M.; Koutsky, L.A.; et al. Head and neck squamous cell cancer and the human papillomavirus: summary of a National Cancer Institute State of the Science Meeting, November 9-10, 2008, Washington, D.C. Head Neck 2009, 31, 1393-1422, doi:10.1002/hed.21269.

9. Laco, J.; Nekvindova, J.; Novakova, V.; Celakovsky, P.; Dolezalova, H.; Tucek, L.; Vosmikova, H.; Vosmik, M.; Neskudlova, T.; Cermakova, E.; et al. Biologic importance and prognostic significance of selected clinicopathological parameters in patients with oral and oropharyngeal squamous cell carcinoma, with emphasis on smoking, protein p16(INK4a) expression, and HPV status. Neoplasma 2012, 59, 398-408, doi:10.4149/neo_2012_052.

10. Laco, J.; Vosmikova, H.; Novakova, V.; Celakovsky, P.; Dolezalova, H.; Tucek, L.; Nekvindova, J.; Vosmik, M.; Cermakova, E.; Ryska, A. The role of high-risk human papillomavirus infection in oral and oropharyngeal squamous cell carcinoma in non-smoking and non-drinking patients: a clinicopathological and molecular study of 46 cases. Virchows Arch 2011, 458, 179187, doi:10.1007/s00428-010-1037-y.

11. Ang, K.K.; Harris, J.; Wheeler, R.; Weber, R.; Rosenthal, D.I.; Nguyen-Tan, P.F.; Westra, W.H.; Chung, C.H.; Jordan, R.C.; Lu, C.; et al. Human papillomavirus and survival of patients with oropharyngeal cancer. N Engl J Med 2010, 363, 24-35, doi:10.1056/NEJMoa0912217.

12. Gillison, M.L.; D'Souza, G.; Westra, W.; Sugar, E.; Xiao, W.; Begum, S.; Viscidi, R. Distinct risk factor profiles for human papillomavirus type 16-positive and human papillomavirus type 16-negative head and neck cancers. J Natl Cancer Inst 2008, 100, 407-420, doi:10.1093/jnci/djn025.

13. Lechner, M.; Frampton, G.M.; Fenton, T.; Feber, A.; Palmer, G.; Jay, A.; Pillay, N.; Forster, M.; Cronin, M.T.; Lipson, D.; et al. Targeted next-generation sequencing of head and neck squamous cell carcinoma identifies novel genetic alterations in HPV+ and HPV- tumors. Genome Med 2013, 5, 49, doi:10.1186/gm453.

14. Dayyani, F.; Etzel, C.J.; Liu, M.; Ho, C.H.; Lippman, S.M.; Tsao, A.S. Meta-analysis of the impact of human papillomavirus (HPV) on cancer risk and overall survival in head and neck squamous cell carcinomas (HNSCC). Head Neck Oncol 2010, 2, 15, doi:10.1186/1758-3284-2-15. 
15. Fakhry, C.; Westra, W.H.; Li, S.; Cmelak, A.; Ridge, J.A.; Pinto, H.; Forastiere, A.; Gillison, M.L. Improved survival of patients with human papillomavirus-positive head and neck squamous cell carcinoma in a prospective clinical trial. J Natl Cancer Inst 2008, 100, 261-269, doi:10.1093/jnci/djn011.

16. Syrjanen, S. Human papillomavirus (HPV) in head and neck cancer. J Clin Virol 2005, 32 Suppl 1, S59-66, doi:10.1016/j.jcv.2004.11.017.

17. El-Naggar, A.K.; Westra, W.H. p16 expression as a surrogate marker for HPV-related oropharyngeal carcinoma: a guide for interpretative relevance and consistency. Head Neck 2012, 34, 459-461, doi:10.1002/hed.21974.

18. Kalof, A.N.; Cooper, K. p16INK4a immunoexpression: surrogate marker of high-risk HPV and high-grade cervical intraepithelial neoplasia. Adv Anat Pathol 2006, 13, 190-194, doi:10.1097/00125480-200607000-00006.

19. Lewis, J.S., Jr.; Thorstad, W.L.; Chernock, R.D.; Haughey, B.H.; Yip, J.H.; Zhang, Q.; El-Mofty, S.K. p16 positive oropharyngeal squamous cell carcinoma:an entity with a favorable prognosis regardless of tumor HPV status. Am J Surg Pathol 2010, 34, 1088-1096, doi:10.1097/PAS.0b013e3181e84652.

20. Yamashita, Y.; Hasegawa, M.; Deng, Z.; Maeda, H.; Kondo, S.; Kyuna, A.; Matayoshi, S.; Agena, S.; Uehara, T.; Kouzaki, H.; et al. Human papillomavirus infection and immunohistochemical expression of cell cycle proteins pRb, p53, and p16(INK4a) in sinonasal diseases. Infect Agent Cancer 2015, 10, 23, doi:10.1186/s13027-015-0019-8.

21. Laco, J.; Sieglova, K.; Vosmikova, H.; Dundr, P.; Nemejcova, K.; Michalek, J.; Celakovsky, P.; Chrobok, V.; Mottl, R.; Mottlova, A.; et al. The presence of high-risk human papillomavirus (HPV) E6/E7 mRNA transcripts in a subset of sinonasal carcinomas is evidence of involvement of HPV in its etiopathogenesis. Virchows Arch 2015, 467, 405-415, doi:10.1007/s00428015-1812-x.

22. Khleif, S.N.; DeGregori, J.; Yee, C.L.; Otterson, G.A.; Kaye, F.J.; Nevins, J.R.; Howley, P.M. Inhibition of cyclin DCDK4/CDK6 activity is associated with an E2F-mediated induction of cyclin kinase inhibitor activity. Proc Natl Acad Sci U S A 1996, 93, 4350-4354, doi:10.1073/pnas.93.9.4350.

23. Ohtani, N.; Yamakoshi, K.; Takahashi, A.; Hara, E. The p16INK4a-RB pathway: molecular link between cellular senescence and tumor suppression. J Med Invest 2004, 51, 146-153, doi:10.2152/jmi.51.146.

24. Larque, A.B.; Hakim, S.; Ordi, J.; Nadal, A.; Diaz, A.; del Pino, M.; Marimon, L.; Alobid, I.; Cardesa, A.; Alos, L. High-risk human papillomavirus is transcriptionally active in a subset of sinonasal squamous cell carcinomas. Mod Pathol 2014, 27, 343-351, doi:10.1038/modpathol.2013.155.

25. Alos, L.; Moyano, S.; Nadal, A.; Alobid, I.; Blanch, J.L.; Ayala, E.; Lloveras, B.; Quint, W.; Cardesa, A.; Ordi, J. Human papillomaviruses are identified in a subgroup of sinonasal squamous cell carcinomas with favorable outcome. Cancer 2009, 115, 2701-2709, doi:10.1002/cncr.24309.

26. Lewis, J.S., Jr. Sinonasal Squamous Cell Carcinoma: A Review with Emphasis on Emerging Histologic Subtypes and the Role of Human Papillomavirus. Head Neck Pathol 2016, 10, 60-67, doi:10.1007/s12105-016-0692-y.

27. Bishop, J.A.; Guo, T.W.; Smith, D.F.; Wang, H.; Ogawa, T.; Pai, S.I.; Westra, W.H. Human papillomavirus-related carcinomas of the sinonasal tract. Am J Surg Pathol 2013, 37, 185-192, doi:10.1097/PAS.0b013e3182698673.

28. Registry, A.C. Annual Report of Cancer Statistics. 2009.

29. Barasch, S.; Mohindra, P.; Hennrick, K.; Hartig, G.K.; Harari, P.M.; Yang, D.T. Assessing p16 Status of Oropharyngeal Squamous Cell Carcinoma by Combined Assessment of the Number of Cells Stained and the Confluence of p16 Staining: A Validation by Clinical Outcomes. Am J Surg Pathol 2016, 40, 1261-1269, doi:10.1097/PAS.0000000000000666. 